\title{
Microvascular Coronary Dysfunction in a Woman With Signs and Symptoms of Myocardial Ischemia but No Obstructive Coronary Artery Disease
}

\author{
Ravi S. Kahlon ${ }^{a}$, Kamlesh Kothawade ${ }^{a}$, Chrisandra Shufelt ${ }^{\mathrm{a}}$, Saibal Kar ${ }^{\mathrm{a}}$, \\ Puja K. Mehta ${ }^{a}$, C. Noel Bairey Merz ${ }^{\text {, b }}$
}

\begin{abstract}
Women with signs and symptoms of myocardial ischemia often have no obstructive coronary artery disease by invasive coronary angiography when compared to men. Microvascular coronary dysfunction (MCD) is thought to be a key contributory mechanism for myocardial ischemia in women with chest pain and no obstructive CAD. We report a case of a 53-year-old post-menopausal female with hyperlipidemia evaluated for persistent chest pain following a non-ST elevation myocardial infarction (NSTEMI) and no obstructive CAD by coronary angiography. Vital signs and physical examination were within normal limits. Prior EKG showed diffuse abnormal $\mathrm{T}$ wave inversion and flattening in anterior and inferior leads. Echocardiography demonstrated an ejection fraction of $72 \%$ with no regional wall motion abnormalities. Laboratory values revealed a positive heterozygote factor V Leiden mutation. Given the constellation of persistent chest pain, no obstructive CAD, and prior NSTEMI, a diagnosis of MCD was suspected and she underwent coronary reactivity testing (CRT) with intracoronary infusions of adenosine, acetylcholine, and nitroglycerin to test non-endothelial and endothelial dependent, micro- and macrovascular coronary function. CRT results were consistent with a diagnosis of MCD. A statin and angiotensin converting enzyme inhibitor was added to her existing treatment of beta-blocker and nitrates. Additionally, she was started on warfarin therapy due to a positive factor $\mathrm{V}$ Leiden in the setting of prior NSTEMI. Her symptoms improved at one-month follow-up and she is currently asymptomatic by selfreport. In women with signs and symptoms of myocardial ischemia and no obstructive $\mathrm{CAD}$, it is important to identify and diagnose $\mathrm{MCD}$, as inadequate diagnosis is associated with an increased risk of adverse cardiovascular events including myocardial infarction,
\end{abstract}

Manuscript accepted for publication January 2, 2013

${ }^{a}$ Barbra Streisand Women's Heart Center, Cedars-Sinai Heart Institute, Los Angeles, CA, USA

${ }^{\mathrm{b}}$ Corresponding author: C. Noel Bairey Merz, Women's Guild

Endowed Chair in Women's Health, Director, Women's Heart Center, Director, Preventive Cardiac Center, Professor of Medicine, Cedars-Sinai Heart Institute, 444 S San Vicente Blvd, Suite 600, Los Angeles, CA 90048, USA. Email: Noel.BaireyMerz@cshs.org

doi: http://dx.doi.org/10.4021/jmc1048w congestive heart failure, and sudden cardiac death. Identification of MCD and clinical awareness are vital for optimal medical therapy and reduced CVD risk. Clinical trials testing efficacy of traditional and novel interventions are needed in MCD populations.

Keywords: Microvascular coronary dysfunction; Coronary reactivity testing; Non-Obstructive coronary artery disease

\section{Introduction}

Patients undergoing coronary angiography for acute coronary syndromes, including unstable angina, ST elevation and non-ST elevation myocardial infarction, often have no obstructive coronary artery disease (CAD) on invasive angiography [1]. There is a higher prevalence of no obstructive $\mathrm{CAD}$ in women compared to men, and women exhibit a greater symptom burden and more functional disability compared to men [2-4]. Microvascular Coronary Dysfunction (MCD) is thought to be a key contributory mechanism for myocardial ischemia in women with chest pain and no obstructive CAD. Identification of angina caused by MCD is crucial due to the associated major adverse cardiac events such as myocardial infarction, congestive heart failure, and sudden cardiac death $[5,6]$. MCD as a cause of angina also contributes to poor quality of life, morbidity, and health care costs [7]. Thus, a thorough evaluation of patients with persistent chest pain is important for accurate diagnosis and treatment. Here, we present the case of a woman with persistent chest pain symptoms following a non-ST elevation myocardial infarction (NSTEMI) and no obstructive CAD documented by angiography. This case highlights the utility of coronary reactivity testing as a diagnostic tool for evaluating cardiac etiologies of persistent chest pain.

\section{Case Report}

A 53-year-old female was referred to a tertiary heart center for a second opinion and further evaluation of persistent episodes of chest pain. Her medical history was significant only for hyperlipidemia on statin therapy. She had no additional 


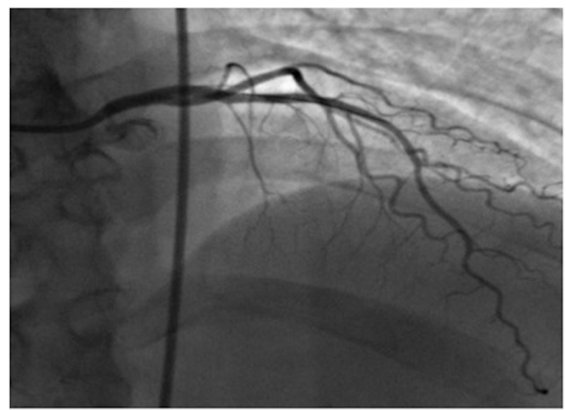

a

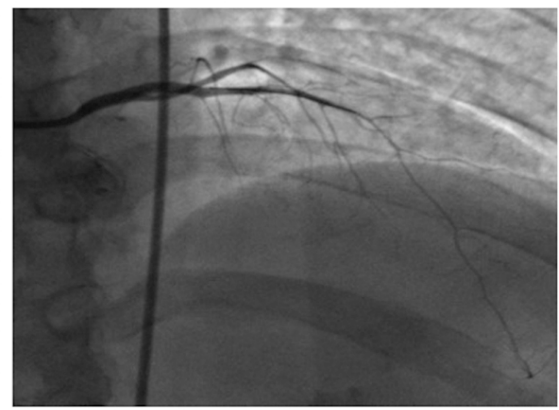

b

Figure 1. Angiogram of left anterior descending (LAD): There was $60 \%$ reduction in LAD diameter measured by Quantitative Coronary Angiography (QCA) following intracoronary injections of $10^{-4}$ acetylcholine (b) from baseline (a) indicating coronary endothelial dysfunction (normal response is vasodilatation). Arrows indicate position of tip of the Doppler flow wire.

cardiac risk factors including no history of diabetes, hypertension, or family history of premature CAD. The patient had quit smoking 29 years prior.

Two months prior to presentation she began experiencing left arm tightness and tingling, for which she was hospitalized at an outside center. Her initial ECG showed T-wave abnormalities in an anterior-inferior distribution, with inverted $\mathrm{T}$ waves in V1 to V6. Troponin on this hospitalization peaked at $6.35 \mathrm{ng} / \mathrm{mL}$ and patient was transferred to an outside facility for further work-up. She underwent coronary angiography that found an LVEDP of $10 \mathrm{mmHg}$, ejection fraction of $60 \%$ with normal wall motion, and open coronary arteries. The patient was discharged home on aspirin $81 \mathrm{mg}$, clopidogrel, and pravastatin. She visited a cardiologist as an outpatient and was started on metoprolol XL.

One month prior to presentation at our facility she experienced a similar episode, with neck and back pain, and subsequently went to her nearest emergency room. Her ECG on this second visit again showed $\mathrm{T}$ wave abnormalities with T-wave inversions in V1 through V3, flattened $\mathrm{T}$ waves in V4 through V6, as well as lead III and aVF. There were no ST elevations. A resting 2-D echo showed no wall motion abnormalities and an EF of $73 \%$. Troponin level peaked on this admission at $5.88 \mathrm{ng} / \mathrm{mL}$ and she was discharged home pain-free and scheduled for outpatient follow-up.

Six days prior to presentation, the patient was driving with her husband when she developed acute onset of neck and squeezing left arm pain with associated nausea, unrelieved by ibuprofen and minimally relieved by sublingual nitroglycerin. The patient ignored the continued pain that persisted, and she was eventually seen at an outside hospital where she was found to have an elevated troponin to approximately $7 \mathrm{ng} / \mathrm{mL}$. Patient was then transferred to a tertiary heart center for further evaluation.

On initial examination she was afebrile, with blood pressure 130/60 $\mathrm{mmHg}$, pulse of $80 \mathrm{bpm}$, respirations 18, and oxygen saturation of $98 \%$ on room air. Physical exam was unremarkable. ECG and 2-D echocardiogram were unchanged from her prior hospitalization. Laboratory values were notable for a positive factor $\mathrm{V}$ Leiden mutation (heterozygote), and an elevated ESR of $27 \mathrm{~mm} / \mathrm{hr}$. Given the constellation of persistent chest pain, absence of obstructive CAD, and prior NSTEMI, a diagnosis of MCD was suspected. Adenosine stress MRI showed no perfusion defects and no wall motion abnormalities. The patient was discharged on her previous medications with the addition of isosorbide mononitrate $60 \mathrm{mg}$ every morning.

She subsequently underwent coronary reactivity testing (CRT) with intracoronary infusions of adenosine, acetylcholine (Ach), and nitroglycerin (NTG) to test non-endothelial and endothelial micro- and macrovascular coronary function (Fig. 1, 2) (Table 1) using previously published methods [8].

Coronary angiography once again demonstrated no evidence of obstructive coronary disease. The right coronary was small with possible scalloping suggestive of luminal irregularities consistent with atherosclerosis. Left ventricular end diastolic filling pressure was elevated at $18 \mathrm{mmHg}$. CRT was markedly abnormal, with an abnormal coronary flow reserve to intracoronary adenosine at 2.3 (normal is greater than 2.5), and abnormal coronary endothelial function response to acetylcholine with $60 \%$ vasoconstriction (normal response is positive dilation). Microvascular endothelial coronary blood flow was calculated to be abnormal at $62 \%$ decrease in response to Ach (normal response is greater than $50 \%$ increase). There was a normal nitroglycerin response, consistent with normal smooth muscle function of $38 \%$ dilation (normal response is greater than 20\%). CRT results were consistent with a diagnosis of MCD. The patient was continued on her beta-blocker and nitrates. Given endothelial dysfunction, an ACE inhibitor and a statin were added to her regimen. Due to her positive factor V Leiden and recurrent NSTEMIs she was transitioned from aspirin to warfarin therapy.

Four months later, the patient presented to a community 


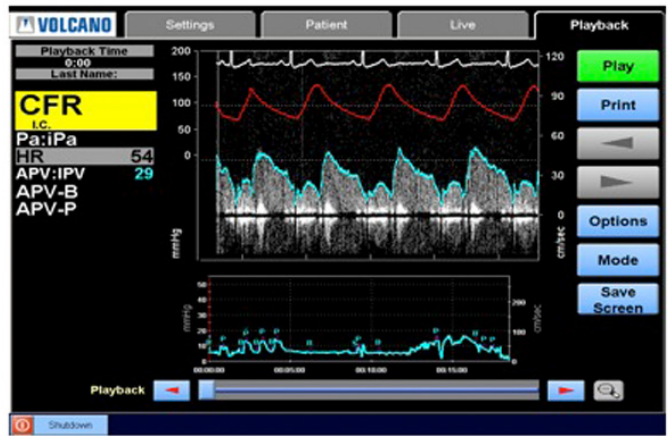

a

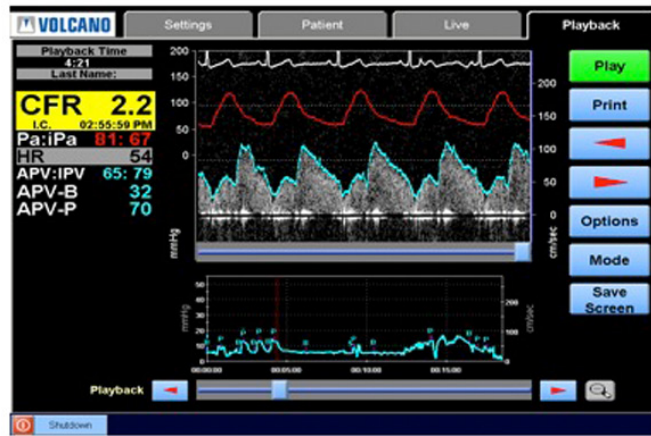

b

Figure 2. Coronary Reactivity Testing measuring peak velocity $\mathrm{cm} / \mathrm{sec}$ and left intracoronary flow reserve (CFR) before adenosine (a) and after infusion of adenosine (b). Normal CFR is $>2.5$. Abnormal CFR in this patient confirms the diagnosis of MCD.

hospital after experiencing nausea and vomiting, and chest pain unrelieved by nitroglycerin. She was found to have a troponin peak of $6.5 \mathrm{ng} / \mathrm{mL}$ and INR of 3.2 on admission. Patient was started on a nitroglycerin drip for her persistent chest pain and elevated troponins. After transfer to a facility providing higher-level care, she became stable and chest pain free. Again troponins were down trending, and vital signs, examination, and laboratory values were within normal limits. The patient underwent repeat coronary angiography that showed no obstructive coronary disease, normal LVEDP (8 $\mathrm{mmHg}$ ), and normal EF (73\%), but was noted to have vasospasm of the right coronary ostium during the procedure with a drop in pressure suggestive of a vasospastic response. Endomyocardial biopsy was performed and was negative for myocarditis or an infiltrative process such as sarcoid or amyloid. Given that vasculitis was a possible cause of persistent troponin elevation, she received a vascular workup, including anticardiolipin IgA and IgM, which were negative, and complement C3, C4 levels, which were within normal lim- its. Similarly, ANA was undetectable. A Russell viper venom and lupus anticoagulant were both negative.

The patient was discharged in stable condition with phase II cardiac rehab and the following changes in her medication regimen. Her beta-blocker was switched to Cardizem given the ostial spasm seen on angiography. She was started on Ranolazine $500 \mathrm{mg}$ twice daily given non-obstructive $\mathrm{CAD}$, presence of $\mathrm{MCD}$, and refractory pain with nitroglycerin. Warfarin was switched back to low-dose aspirin given that her heterozygous factor $\mathrm{V}$ Leiden had not produced a thrombotic event, and the coronary protective effects of aspirin. The patient's symptoms were improved at 1-month follow-up, and she is currently asymptomatic by self- report.

\section{Discussion}

The etiology of persistent chest pain is not only difficult,

Table 1. The Results of the Coronary Reactivity Testing

\begin{tabular}{lll}
\hline & Endothelial Dependant & Non-endothelial Dependant \\
\hline Microvascular Dysfunction & $\begin{array}{l}\text { Coronary Blood Flow to Ach } \\
\text { Abnormal }\end{array}$ & $\begin{array}{l}\text { CFR to Adenosine } \\
\text { Abnormal }\end{array}$ \\
Macrovascular Dysfunction & $\begin{array}{l}\text { QCA to Ach } \\
\text { Abnormal }\end{array}$ & $\begin{array}{l}\text { QCA to NTG } \\
\text { Normal }\end{array}$ \\
\hline
\end{tabular}

Normal values include: Coronary Blood Flow (CBF) to Acetylcholine (Ach) $>50 \%$, Intracoronary Flow Reserve (CFR) to Adenosine > 2.5, Quantitative Coronary Angiography (QCA) to Acetylcholine > 5\% change, QCA to Nitroglycerin (NTG) > $20 \%$. CRT was performed by infusing vasoactive substances through a guiding catheter placed in the left main coronary artery. Doppler guide-wire (0.014-inch diameter, FloWire, JOMED/Cardiometrics/Volcano, San Diego, California) was positioned in the proximal left anterior descending coronary artery. 
but also encompasses a broad array of diagnoses. Too often, once coronary angiography reveals normal or open coronary arteries and patients are usually reassured and discharged with the diagnosis of non-cardiac chest pain without further cardiac evaluation. This can lead to adverse effects on quality of life, morbidity, and cost of healthcare. Eighty six percent of patients with continued chest pain and no evidence of CAD were found to have chest pain at least weekly for up to one year post-angiogram, with unchanged or even worsening pain, and with half reporting functional disability including limitations on activities of daily living and/or inability to work $[9,10]$. Despite open coronary angiograms, twenty to fifty percent of patients are re-hospitalized for chest pain with an average lifetime cost per patient set at approximately eight hundred thousand dollars [7].

Approximately 50\% of women with symptoms of persistent chest pain, evidence of ischemia, and presence of no obstructive CAD have been found to have MCD [11]. The Women's Ischemia Syndrome Evaluation (WISE) study group has shown that MCD is prevalent in women with the triad of chest pain, evidence of ischemia by stress testing, and non-obstructive CAD [12]. These women tend to be younger with mean ages in the fifth decade of life (range 21 - 86 years) $[2-4,6]$. The diagnosis of MCD is challenging because the coronary microvasculature is not directly visualized during routine coronary angiography. It is the microvasculature however, which is responsible for coronary blood flow regulation and therefore oxygen delivery. Autoregulation involves both endothelial dependent and independent mechanisms. Coronary endothelial dysfunction is an indicator of early atherosclerosis [13] and is independently associated with cardiovascular events $[5,6]$. Patients with coronary endothelial dysfunction and non-obstructive CAD have an increased risk of fatal and non-fatal major adverse cardiac events including sudden cardiac death, myocardial infarction, and congestive heart failure $[5,6,8]$. The above case illustrates that although the patient had no detectable ischemia on stress testing, this likely reflects the low sensitivity of the diagnostic modality and not the absence of ischemia, and the cause of her persistent chest pain is, at least in part, due to MCD.

During CRT four measures of coronary vascular autoregulation are assessed: 1) coronary flow reserve (CFR) in response to intracoronary administration of adenosine as an indicator of non-endothelial microvascular function [14]; 2) coronary blood flow (CBF) indicative of endothelial microvascular function; 3) coronary artery diameter in response to intracoronary administration of acetylcholine as measured by quantitative coronary angiography (QCA) an indicator of endothelial macrovascular function; and 4) coronary artery diameter in response to intracoronary nitroglycerin as measured by QCA indicative of non-endothelial macrovascular function. Data regarding the safety of CRT has found that this diagnostic modality is at least equal to routine coronary angiogram with approximately 1 in 1,000 risk of serious adverse events $[15,16]$. Moreover, because the risk of fatal and non-fatal major adverse cardiac events associated with endothelial dysfunction $[5,6,8,17]$, and $\operatorname{MCD}[17,18]$ is high (up to $2.5 \%$ ) compared with the risk of serious and/or adverse events with CRT $[15,16](0.7 \%)$, patients with the triad of persistent chest pain, objective evidence of myocardial ischemia, and no obstructive CAD should be considered for CRT when the diagnosis is uncertain.

Treatment of MCD can also be challenging due to a lack of universally accepted diagnostic criteria and multiple pathways contributing to the pathophysiology. Goals, as with therapy for obstructive CAD, include controlling debilitating symptoms, improving quality of life, reducing hospitalization incidence and repeat testing, and improving survival. Cardiac rehabilitation can be initiated to minimize symptoms and has been shown to be effective in these patients in increasing exercise capacity and symptom relief [19]. Statins may improve endothelial function by lipid-independent mechanisms through their anti-inflammatory and anti-oxidant properties, or like ACEIs and ARBs, through their ability to restore vascular nitric oxide and thus endothelial-dependent relaxation of coronary resistance vessels [20-22]. Beta blockade, particularly with atenolol, has been shown to reduce the number and severity of anginal episodes and improve functional capacity in patients with MCD [23]. Additional benefit may be seen from agents with alpha-blockade, such as carvedilol, in patients with coronary spasm [24]. Recently, a pilot study of Ranolazine, an anti-anginal agent that reduces calcium flow in myocytes through inhibition of the late sodium current, has been shown to improve angina in women with evidence of ischemia but no obstructive CAD [25]. While there are no clinical trials regarding the role of nitrates in patients with $\mathrm{MCD}$, at least observationally, their effects on angina frequency and duration can be unpredictable in these patients. Similarly, the use of calcium channel blockers for management of MCD is not yet supported by evidence, although it is first line therapy for vasospastic angina and has been shown to improve exercise capacity and chest pain in patients with no obstructive CAD and limited vasodilatory reserve [26].

In conclusion, this case highlights the clinical importance and challenges of diagnosing MCD. In women with signs and symptoms of myocardial ischemia, NSTEMI, and no obstructive CAD, MCD may be a mechanism leading to symptoms. It is therefore important to identify and diagnose in the appropriate clinical setting, as MCD is associated with an increased risk of adverse cardiovascular events. Moreover, MCD is the presumable cause of symptoms that can lead to repeated testing, hospitalization, and disability, creating substantial economic, physical, and emotional hardships [7]. It is imperative that clinicians are aware of this condition, as early identification of MCD by CRT in the appropriate clinical setting may be beneficial in prognostication and/ or stratification of these patients for optimal medical therapy. 


\section{Grant Support}

This work was supported by contracts from the National Heart, Lung and Blood Institute, nos. N01-HV-68161, N01-HV-68162, N01-HV-68163, N01-HV-68164, grants U0164829, U01 HL649141, U01 HL649241, T32HL69751, 1R03AG032631 from the National Institute on Aging, GCRC grant MO1-RR00425 from the National Center for Research Resources, NIH CTSI Grant UL1TR000124 and grants from the Gustavus and Louis Pfeiffer Research Foundation, Danville, NJ, The Women's Guild of Cedars-Sinai Medical Center, Los Angeles, CA, The Ladies Hospital Aid Society of Western Pennsylvania, Pittsburgh, PA, and QMED, Inc., Laurence Harbor, NJ, the Edythe L. Broad Women's Heart Research Fellowship, Cedars-Sinai Medical Center, Los Angeles, California, and the Barbra Streisand Women's Cardiovascular Research and Education Program, Cedars-Sinai Medical Center, Los Angeles.

\section{Disclosures}

There are no relevant conflicts of interest of any of the authors to disclose.

\section{References}

1. Jespersen L, Hvelplund A, Abildstrom SZ, Pedersen F, Galatius S, Madsen JK, Jorgensen E, et al. Stable angina pectoris with no obstructive coronary artery disease is associated with increased risks of major adverse cardiovascular events. Eur Heart J. 2012;33(6):734-744.

2. Diver DJ, Bier JD, Ferreira PE, Sharaf BL, McCabe C, Thompson B, Chaitman B, et al. Clinical and arteriographic characterization of patients with unstable angina without critical coronary arterial narrowing (from the TIMI-IIIA Trial). Am J Cardiol. 1994;74(6):531-537.

3. Hochman JS, Tamis JE, Thompson TD, Weaver WD, White HD, Van de Werf F, Aylward P, et al. Sex, clinical presentation, and outcome in patients with acute coronary syndromes. Global Use of Strategies to Open Occluded Coronary Arteries in Acute Coronary Syndromes IIb Investigators. N Engl J Med. 1999;341(4):226-232.

4. Phibbs B, Fleming T, Ewy GA, Butman S, Ambrose J, Gorlin R, Orme E, et al. Frequency of normal coronary arteriograms in three academic medical centers and one community hospital. Am J Cardiol. 1988;62(7):472-474.

5. Halcox JP, Schenke WH, Zalos G, Mincemoyer R, Prasad A, Waclawiw MA, Nour KR, et al. Prognostic value of coronary vascular endothelial dysfunction. Circulation. 2002;106(6):653-658.

6. Suwaidi JA, Hamasaki S, Higano ST, Nishimura RA, Holmes DR, Jr., Lerman A. Long-term follow-up of pa- tients with mild coronary artery disease and endothelial dysfunction. Circulation. 2000;101(9):948-954.

7. Shaw LJ, Merz CN, Pepine CJ, Reis SE, Bittner V, Kip $\mathrm{KE}$, Kelsey SF, et al. The economic burden of angina in women with suspected ischemic heart disease: results from the National Institutes of Health--National Heart, Lung, and Blood Institute--sponsored Women's Ischemia Syndrome Evaluation. Circulation. 2006;114(9):894-904.

8. von Mering GO, Arant CB, Wessel TR, McGorray SP, Bairey Merz CN, Sharaf BL, Smith KM, et al. Abnormal coronary vasomotion as a prognostic indicator of cardiovascular events in women: results from the National Heart, Lung, and Blood Institute-Sponsored Women's Ischemia Syndrome Evaluation (WISE). Circulation. 2004;109(6):722-725.

9. Lantinga LJ, Sprafkin RP, McCroskery JH, Baker MT, Warner RA, Hill NE. One-year psychosocial follow-up of patients with chest pain and angiographically normal coronary arteries. Am J Cardiol. 1988;62(4):209-213.

10. Potts SG, Bass CM. Psychological morbidity in patients with chest pain and normal or near-normal coronary arteries: a long-term follow-up study. Psychol Med. 1995;25(2):339-347.

11. Reis SE, Holubkov R, Conrad Smith AJ, Kelsey SF, Sharaf BL, Reichek N, Rogers WJ, et al. Coronary microvascular dysfunction is highly prevalent in women with chest pain in the absence of coronary artery disease: results from the NHLBI WISE study. Am Heart J. 2001;141(5):735-741.

12. Quyyumi AA. Women and ischemic heart disease: pathophysiologic implications from the Women's Ischemia Syndrome Evaluation (WISE) Study and future research steps. J Am Coll Cardiol. 2006;47(3 Suppl):S66-71.

13. Zeiher AM, Drexler H, Wollschlager H, Just H. Endothelial dysfunction of the coronary microvasculature is associated with coronary blood flow regulation in patients with early atherosclerosis. Circulation. 1991;84(5):1984-1992.

14. Noel Bairey Merz C, Eteiba W, Pepine C, Johnson B, Shaw L, Kelsey S. Cardiac syndrome X: Relation to microvascular angina and other conditions. 2007:167-75.

15. Wei J, Mehta PK, Johnson BD, Samuels B, Kar S, Anderson RD, Azarbal B, et al. Safety of coronary reactivity testing in women with no obstructive coronary artery disease: results from the NHLBI-sponsored WISE (Women's Ischemia Syndrome Evaluation) study. JACC Cardiovasc Interv. 2012;5(6):646-653.

16. Tio RA, Monnink SH, Amoroso G, Jessurun GA, Veeger N, Volkers C, Hautvast R, et al. Safety evaluation of routine intracoronary acetylcholine infusion in patients undergoing a first diagnostic coronary angiogram. J Investig Med. 2002;50(2):133-139.

17. Bairey Merz CN, Shaw LJ, Reis SE, Bittner V, Kelsey $\mathrm{SF}$, Olson M, Johnson BD, et al. Insights from the NHL- 
BI-Sponsored Women's Ischemia Syndrome Evaluation (WISE) Study: Part II: gender differences in presentation, diagnosis, and outcome with regard to genderbased pathophysiology of atherosclerosis and macrovascular and microvascular coronary disease. J Am Coll Cardiol. 2006;47(3 Suppl):S21-29.

18. Gulati M, Cooper-DeHoff RM, McClure C, Johnson BD, Shaw LJ, Handberg EM, Zineh I, et al. Adverse cardiovascular outcomes in women with nonobstructive coronary artery disease: a report from the Women's Ischemia Syndrome Evaluation Study and the St James Women Take Heart Project. Arch Intern Med. 2009;169(9):843850.

19. Eriksson BE, Tyni-Lenne R, Svedenhag J, Hallin R, Jensen-Urstad K, Jensen-Urstad M, Bergman K, et al. Physical training in Syndrome $\mathrm{X}$ : physical training counteracts deconditioning and pain in Syndrome X. J Am Coll Cardiol. 2000;36(5):1619-1625.

20. Bonetti PO, Lerman LO, Lerman A. Endothelial dysfunction: a marker of atherosclerotic risk. Arterioscler Thromb Vasc Biol. 2003;23(2):168-175.

21. Hinoi T, Tomohiro Y, Kajiwara S, Matsuo S, Fujimoto Y, Yamamoto S, Shichijo T, et al. Telmisartan, an angiotensin II type 1 receptor blocker, improves coronary microcirculation and insulin resistance among essential hypertensive patients without left ventricular hypertrophy. Hypertens Res. 2008;31(4):615-622.

22. Tiefenbacher CP, Friedrich S, Bleeke T, Vahl C, Chen X, Niroomand F. ACE inhibitors and statins acutely improve endothelial dysfunction of human coronary arterioles. Am J Physiol Heart Circ Physiol. 2004;286(4):H1425-
1432.

23. Lanza GA, Colonna G, Pasceri V, Maseri A. Atenolol versus amlodipine versus isosorbide-5-mononitrate on anginal symptoms in syndrome X. Am J Cardiol. 1999;84(7):854-856, A858.

24. Anderson JL, Adams CD, Antman EM, Bridges CR, Califf RM, Casey DE, Jr., Chavey WE, 2nd, et al. ACC/ AHA 2007 guidelines for the management of patients with unstable angina/non ST-elevation myocardial infarction: a report of the American College of Cardiology/American Heart Association Task Force on Practice Guidelines (Writing Committee to Revise the 2002 Guidelines for the Management of Patients With Unstable Angina/Non ST-Elevation Myocardial Infarction): developed in collaboration with the American College of Emergency Physicians, the Society for Cardiovascular Angiography and Interventions, and the Society of Thoracic Surgeons: endorsed by the American Association of Cardiovascular and Pulmonary Rehabilitation and the Society for Academic Emergency Medicine. Circulation. 2007;116(7):e148-304.

25. Mehta PK, Goykhman P, Thomson LE, Shufelt C, Wei J, Yang Y, Gill E, et al. Ranolazine improves angina in women with evidence of myocardial ischemia but no obstructive coronary artery disease. JACC Cardiovasc Imaging. 2011;4(5):514-522.

26. Cannon RO, 3rd, Watson RM, Rosing DR, Epstein SE. Efficacy of calcium channel blocker therapy for angina pectoris resulting from small-vessel coronary artery disease and abnormal vasodilator reserve. Am J Cardiol. $1985 ; 56(4): 242-246$. 